Pacific Journal of Mathematic 


\title{
ON THE HITCHCOCK DISTRIBUTION PROBLEM
}

\author{
MERRILL M. FLoOd
}

1. Introduction. Frank L. Hitchcock [1] has offered a mathematical formulation of the problem of determining the most economical manner of distribution of a product from several sources of supply to numerous localities of use, and has suggested a computational procedure for obtaining a solution of his system in any particular case. L. Kantorovitch [2], Tjalling C. Koopmans [3], George B. Dantzig [4b], C. B. Tompkins [5], Julia Robinson [7; 8], Alex Orden [6], and others [4] have also discussed the computational aspects of this problem; paper [5] illustrates the use of the "projection method," due to C. B. Tompkins, as a computational process applicable to either of the Fundamental Problems of the present paper.

We shall be concerned only with the mathematical justification of computational procedure, and shall limit our attention to one specific method of solution of general validity. No attempt will be made to compare the various methods already proposed, either as to their mathematical similarity or as to their relative efficiency in any particular case.

2. The problem. The problem is to find a set of values of the $m n$ variables $x_{i j}$, subject to the following conditions:

$$
\begin{gathered}
\sum_{i=1}^{m} x_{i j}=c_{j}, \sum_{j=1}^{n} x_{i j}=r_{i}, \\
x_{i j} \geq 0,
\end{gathered}
$$

Received January 25, 1952. The author's interest in the problem was aroused by papers on transportation theory presented by Koopmans [4a] and Dantzig [4b] at a conference on linear programming in Chicago during June, 1949, under the auspices of the Cowles Commission for Research in Economics of the University of Chicago. Several other papers presented at this conference are of closely related interest. Professor Koopmans, in his Introduction io the Conference Proceedings [4], also discussed the background and interrelationship of the conference papers-including the bearing of some of these on the Hitchcock distribution problem. The results of the present paper have been presented in three seminar lectures: once in December, 1949, at The RAND Corporation in Santa Monica, once in July, 1950, at the Institute for Numerical Analysis of the National Bureau of Standards in Los Angeles, and once in June, 1951, at the National Bureau of Standards in Washington, D.C. The author is especially indebted to Dr. D. R. Fulkerson, who has given real assistance in simplifying notation and proofs of theorems, for a careful reading of the manuscript.

Pacific J. Math. 3 (1953), 369-386 


$$
\sum_{i, j} x_{i j} d_{i j}=\text { minimum }
$$

The numbers $m, n, r_{i}, c_{j}$, and $d_{i j}$ are given positive integers with $\sum c_{j}=\sum r_{i}$. The indices $i$ and $j$ are understood always to range over these same integers $m$ and $n$, respectively; it is also assumed, for convenience, that $m \geq n$. Any set of values $x_{i j}$ that satisfies all these conditions is called a solution of the problem.

There is no loss of generality in assuming that the $d_{i j}$ are positive integers, rather than rational numbers, since the problem is essentially unchanged if $d_{i j}$ is replaced by $a d_{i j}+b$, where $a$ and $b$ are any positive rational numbers. We have not examined the case in which some of the quantities $r_{i}, c_{j}$, and $d_{i j}$ are irrational. The only effect of irrationality on the results of the present paper is a possible lack of convergence of the iterative process of solution. These considerations are not of importance in the usual applications.

It will sometimes be more convenient to use an alternative statement of the problem, in matrix notation, as follows:

$$
\begin{gathered}
M^{\prime} y \geq b, \\
y \geq 0, \\
a^{\prime} y=\text { minimum. }
\end{gathered}
$$

It is easily seen that the two formulations are equivalent if $y, a, b$, and $M^{\prime}$ are defined as follows:

$$
\begin{aligned}
& y_{n(i-1)+j}=x_{i j}, \\
& a_{n(i-1)+j}=d_{i j}, \\
& M^{\prime}=\left\|\begin{array}{rrrr}
I_{n} & I_{n} & \cdots & I_{n} \\
-I_{n} & -I_{n} & \cdots & -I_{n} \\
J_{1} & J_{2} & \cdots & J_{m} \\
-J_{1} & -J_{2} & \cdots & -J_{m}
\end{array}\right\|, \quad b=\left\|\begin{array}{r}
c \\
-c \\
r \\
-r
\end{array}\right\| \text {, }
\end{aligned}
$$

where $I_{n}$ is the identity matrix of order $n$, and $J_{i}$ is the $m \times n$ matrix with all elements zero except for the $i$ th row in which each element is unity. Of course, $y, a, c$, and $r$ are column matrices (or vectors) with components $y_{n(i-1)+j}$, $a_{n(i-1)+j}, c_{j}$, and $r_{i}$, respectively, and a prime denotes the transpose of a matrix (or vector). 
3. Fundamental theorems. There are several fundamental theorems concerning systems of linear inequalities that are useful for this paper. I reproduce their statements here in a form given by A. W. Tucker in an unpublished note dated December, 1949. The interested reader can find proofs of these theorems, and of others of similar type, in a paper by Gale, Kuhn, and Tucker [4c].

Fundamental Problems. (Here lower case roman letters denote onecolumn vectors, while capitals denote rectangular matrices; $M, a$, and $b$ are given, but $d$ is to be determined.)

PROBLEM I. To satisfy the constraints $M x \leq a, x \geq 0$, and make $b^{\prime} x=d$ for $d$ maximal in the sense that no $x$ satisfying the constraints makes $b^{\prime} x>d$.

PrовLEM II. To satisfy the constraints $M^{\prime} y \geq b, y \geq 0$, and make $a^{\prime} y=d$ for $d$ minimal in the sense that no $y$ satisfying the constraints makes $a^{\prime} y<d$.

Problems I and II are said to be dual.

Fundamental FEAsibility Theorem. The constraints in a problem are feasible (that is, satisfied by some $x$ or $y$ ) if and only if the dual problem in homogeneous form (that is, with $b=0$ or $a=0$ ) has a null solution.

Fundamental Existence TheOREM. i. The vectors $x$ and $y$ are solutions of Problems I and II if and only if they satisfy their constraints in the two problems and make $a^{\prime} y=b^{\prime} x$. Such $x$ and $y$ exist if the constraints in both problems are feasible.

ii. A problem has a solution if and only if its constraints are feasible and its homogeneous form has a null solution.

Fundamental Duality Theorem. A problem has a solution (for a unique $d$ ) if and only if the dual problem has a solution (for the same $d$ ).

4. The dual and combined problems. We note that the problem, as stated in relations (2.4) $-(2.6)$, is a Fundamental Problem of form II. The dual problem is:

$$
\begin{gathered}
M x \leq a \\
x \geq 0 \\
b^{\prime} x=\text { maximum. }
\end{gathered}
$$

This can be rewritten in a more convenient form, for our present purposes, as 
follows:

$$
\begin{gathered}
v_{j}-u_{i} \leq d_{i j}, \\
\sum c_{j} v_{j}-\sum r_{i} u_{i}=\operatorname{maximum},
\end{gathered}
$$

where $v_{j}=x_{j}-x_{n+j}$, and $u_{i}=-x_{2 n+i}+x_{2 n+m+i}$; we omit the condition (4.2), that $x \geq 0$, since this imposes no limitation on $u_{i}$ and $v_{j}$.

THEOREM 1. The problem has a solution.

Proof. By the Fundamental Existence Theorem, there is a solution if and only if the constraints are feasible and $y=0$ is a solution of the problem when $b=0$. Now

$$
\sum_{c_{j}}=\sum_{r_{i}}
$$

so

$$
x_{i j}=\frac{r_{i} c_{j}}{\sum r_{i}}
$$

satisfies the constraints. When $b=0$, obviously the only values that satisfy the constraints are $x_{i j}=0$, and so the theorem is proved.

By the Fundamental Duality Theorem, we see:

COROLLARY 1A. The dual problem has a solution.

THEOREM 2. The numbers $x_{i j}$, and $u_{i}, v_{j}$, are solutions of the problem and the dual, respectively, if and only if they satisfy:

$$
\sum_{j} x_{i j}=r_{i}, \quad \sum_{i} x_{i j}=c_{j}, \quad x_{i j} \geq 0,
$$

$$
d_{i j}+u_{i}-v_{j} \geq 0
$$

$$
x_{i j}\left(d_{i j}+u_{i}-v_{j}\right)=0 \text {. }
$$

Proof. Since (4.6) and (4.7) are simply the constraints for the problem and the dual, respectively, it remains only to show that $(4.8)$ is equivalent to the condition $a^{\prime} y-b^{\prime} x=0$. Now 


$$
\begin{aligned}
a^{\prime} y-b^{\prime} x & =\sum_{i, j} x_{i j} d_{i j}-\sum_{j} c_{j} v_{j}+\sum_{i} r_{i} u_{i} \\
& =\sum_{i, j} x_{i j} d_{i j}-\sum_{i, j} x_{i j} v_{j}+\sum_{i, j} x_{i j} u_{i}=\sum_{i, j} x_{i j}\left(d_{i j}+u_{i}-v_{j}\right) .
\end{aligned}
$$

Since each term in this sum is nonnegative,

$$
a^{\prime} y-b^{\prime} x=0
$$

if and only if

$$
x_{i j}\left(d_{i j}+u_{i}-v_{j}\right)=0 \text {. }
$$

We refer to the problem of finding values for $x_{i j}, u_{i}$, and $v_{j}$ that satisfy (4.6)-(4.8) as the "combined problem", and note that the combined problem always has a solution.

5. Linear graphs. It will be convenient, for some purposes, to associate linear graphs [9] with certain subsets of the elements of a matrix $S=\left\|s_{h k}\right\|$. If $I$ is a given subset of the elements of $S$, we define the $I$-graph $L$ of $S$ as follows: the vertices of $L$ are all the points $(h, k)$ in the Cartesian plane for which $s_{h k} \in I$; the arcs of $L$ are all line-segments joining pairs of neighboring vertices with either equal abscissas or equal ordinates, where two vertices with equal abscissas (ordinates) are neighboring if they are not separated by another vertex of $L$ with the same abscissa (ordinate). For the moment, denote the vertices of $L$ by symbols $a, b, c, \cdots, f$, and the arcs by symbols such as $a b, b c, \cdots, c f$ (no distinction is made between the arcs $a b$ and $b a$ ). Then a chain is a set of one or more distinct arcs that can be arranged as $a b, b c, \cdots, d e$, $e f$, where vertices denoted by different symbols are distinct. A cycle is a set of distinct arcs (at least four are necessary) that can be ordered as $a b, b c, \cdots, e f$, $\mathrm{fa}$, the vertices being distinct as in the case of a chain. A graph is connected if each pair of vertices is joined by a chain. A forest is a graph containing no cycles, and a tree is a connected forest.

If $L$ contains $v$ vertices, $a$ arcs, and $p$ connected pieces, the number $\mu=a-v+p$ is known as the cyclomatic number (or first Betti number) of $L$. It follows from a well-known theorem [9] concerning linear graphs in general that: (i) $L$ is a forest if and only if $\mu=0$, and (ii) $L$ contains just one cycle if and only if $\mu=1$.

Note that $L$ contains a cycle if and only if there is a subset of $I$ that can be arranged as a sequence 


$$
s_{h_{1} k_{1}}, s_{h_{1} k_{2}}, s_{h_{2} k_{2}}, s_{h_{2} k_{3}}, \ldots, s_{h_{\sigma}} k_{\sigma}, s_{h_{\sigma} k_{1}} \text {, }
$$

where the $h$ 's and $k$ 's are distinct among themselves; and $L$ contains a single cycle if and only if $I$ contains just one subset that can be arranged in the displayed form. We call such a subset of $I$ an $I$-circuit on $S$, and denote it by $\left[S_{\sigma}\right]$. For a particular arrangement of $\left[S_{\sigma}\right]$, we also refer to the terms $s_{h_{\alpha}} k_{\alpha}$ as oddterms, the others as even-terms.

In case $I$ consists of all $s_{h k}>0$, as it frequently will, we speak of the positive graph of $S$, positive circuits on $S$, and abbreviate such statements as "the positive graph of $S$ is a forest" to " $S$ is a forest".

6. The method of solution. In the method of solution to be developed for the problem, we start with a special set of values $X^{0}=\left\|x_{i j}^{0}\right\|$ that satisfy the constraints (4.6). We then test to determine whether or not there exist $u_{i}$ and $v_{j}$ satisfying the relations (4.7) and (4.8) for the given $X^{0}$. If so, then $X^{0}$ is a solution, otherwise not. The method next yields a new trial matrix $X^{1}=\left\|x_{i j}^{1}\right\|$, if $X^{0}$ is not a solution, such that

$$
\sum_{i, j}\left(x_{i j}^{0}-x_{i j}^{1}\right) d_{i j} \geq 1 .
$$

After a finite number of steps this process necessarily must terminate, and it leads to an exact integral solution of the problem.

The first trial matrix $X^{0}$ is a forest of $t$ trees, and has $m+n-t$ nonzero elements. According as $t=1$ or $t>1$, two essentially different cases may be met at each stage of the solution process. ${ }^{1}$

At each stage when $X=\left\|x_{i j}\right\|$ is a tree, the equations (4.8) have a general solution for $u_{i}$ and $v_{j}$ with one free parameter, say $u_{1}$. However, the quantities $d_{i j}+u_{i}-v_{j}$ are uniquely determined in this case, so it is sufficient to calculate them and note whether or not they are all nonnegative in order to decide whether or not $X$ is a solution. If some

${ }^{1}$ These are the nondegenerate and degenerate cases in the work of Dantzig [4b]. We shall use these terms also. The method of solution developed by Dantzig [4b] for the nondegenerate case is essentially the same as the one in the present paper, although the derivations of the results are quite different. Orden [6] has subsequently given an elegant method for reducing the degenerate case to the nondegenerate one, as an extension of the $\epsilon$-method proposed by Dantzig [4b]. The author believes that the treatment of the degenerate case provides the only results in the present paper that are new, or at least fresh for the Hitchcock problem, and also of some mathematical interest. It also seems likely that the method given here will often be more efficient computationally, in the degenerate case, than the Dantzig-Orden $\epsilon$-method. 


$$
d_{i_{1} j_{1}}+u_{i_{1}}-v_{j_{1}}<0
$$

then there is a unique $l$-circuit $\left[X_{s}\right]$ on $X$, where $I$ consists of $x_{i_{1} j_{1}}$ and all positive $x_{i j}$, that may be arranged with $x_{i_{1} j_{1}}$ as the second term, say. Let $g$ denote the smallest odd-term of $\left[X_{s}\right]$. Then the new trial matrix $X^{*}$ is obtained from $X$ by adding $g$ to the even terms of $\left[X_{s}\right]$, subtracting $g$ from the odd-terms, and leaving the other elements of $X$ unchanged.

At each stage when $X$ is a forest of $t>1$ trees, the equations (4.8) have a general solution for $u_{i}$ and $v_{j}$ with $t$ independent parameters, and the quantities $d_{i j}+u_{i}-v_{j}$ involve $t-1$ independent parameters. The rows and columns of the matrix $X$ are rearranged so that it can be represented as a square matrix of order $t$ whose $t^{2}$ elements are submatrices $X_{a b}$ such that $X_{a b}=0$ if $a \neq b$, and $X_{a a}$ is a tree with $m_{a}+n_{a}-1$ nonzero elements and is of order $m_{a} \times n_{a}$. It may also be assumed that each $X_{a a}$ is a solution of its subproblem. We can select

$$
u_{1}, u_{m_{1}+1}, \cdots, u_{m_{1}}+\cdots+m_{t-1}+1
$$

to be the $t$ parameters. If we assign these the value zero and denote this particular solution of $(4.8)$ by $\bar{u}_{i}$ and $\bar{v}_{j}$, then we may define numbers

$$
\bar{p}_{i j}=d_{i j}+\bar{u}_{i}-\bar{v}_{j}
$$

We partition the matrix $\bar{P}=\left\|\bar{p}_{i j}\right\|$ into submatrices corresponding to the $X_{a b}$ and denote them $\bar{P}_{a b}$. Let $p_{a b}$ be the smallest element in $\bar{P}_{a b}$ and define the square matrix $P$ of order $t$ by $P=\left\|p_{a b}\right\|$. To designate the position of $p_{a b}$ in the matrix $\bar{P}=\left\|\bar{p}_{i j}\right\|$, we may write $p_{a b}$ alternatively as

$$
\bar{p}_{a b}^{i_{a} j_{b}}
$$

the subscripts referring to the submatrix and the superscripts to the rows and columns in the submatrix. When it introduces no ambiguity, the subscripts on the superscripts will be omitted in order to simplify the notation.

The test as to whether or not $X$ is a solution consists of forming all sums

$$
p_{a_{1} a_{2}} \cdots a_{h}=p_{a_{1} a_{2}}+p_{a_{2} a_{3}}+\cdots+p_{a_{h} a_{1}}
$$

for $h=2,3, \cdots, t$, where $\left(a_{1} a_{2} \cdots a_{h}\right)$ is any permutation of $h$ different positive integers, none greater than $t ; X$ is a solution if and only if all such sums are nonnegative. 
If any

$$
p_{a_{1} a_{2} \cdots a_{h}}<0
$$

then there is a unique $I$-circuit $\left[X_{s}\right]$ on $X$, where $I$ consists of all positive $x_{i j}$ together with all $x_{i j}$ that correspond to the terms

$$
\bar{p}_{a_{k}}^{i} a_{k+1}^{j} \text { of } p_{a_{1} a_{2} \cdots a_{h}}
$$

which can be arranged to involve all

$$
x_{a_{k}}^{i} a_{k+1}^{j}
$$

as even-terms. If $g$ is the smallest odd-term in $\left[X_{s}\right]$, then (as in the nondegenerate case) the new trial matrix $X^{*}$ is obtained by adding $g$ to the even-terms of $\left[X_{s}\right]$, subtracting $g$ from the odd-terms, and leaving the other elements of $X$ unchanged.

7. The initial trial solution. An $X$ that satisfies (4.6) will be called a trial solution. It would be all right to take the positive values

$$
\frac{r_{i} c_{j}}{\sum r_{i}}
$$

for the initial trial solution $X^{0}=\left\|x_{i j}^{0}\right\|$. An alternative is to construct an initial trial solution that is a forest. It is always possible to do this in integral values. The following theorem certifies the existence of such an integral trial solution. The method of proof shows how to construct one.

THEOREM 3. There is a matrix $X^{0}=\left\|x_{i j}^{0}\right\|$ with integral elements that satisfies (4.6) and is a forest.

Proof. The theorem is trivial for $m=1$. Assume the theorem is true for $m$ and consider the case $m+1$.

Let the notation be chosen so that

$$
r_{1} \geq r_{2} \geq \cdots \geq r_{m+1}>0, \text { and } c_{1} \geq c_{2} \geq \cdots \geq c_{n}>0
$$

If $n<m+1$, then $c_{1}>r_{m+1}$. If $n=m+1$ then $c_{1}>r_{m+1}$ unless $c_{i}=r_{j}=\lambda$ 
(for all $i$ and $j$ ); in this latter case $X^{0}=\lambda$ satisfies the conditions of the theorem. Hence, by the induction hypothesis, there is a set of nonnegative integers $x_{i j}^{*}(i=1, \cdots, m)$ such that

$$
\sum_{i} x_{i j}^{*}=c_{j}-\delta_{1 j} r_{m+1}, \sum_{j} x_{i j}^{*}=r_{i}, \text { and } X^{*}=\left\|x_{i j}^{*}\right\|
$$

is a forest. Then $X^{0}$, defined by

$$
x_{i j}^{0}=x_{i j}^{*}, \quad x_{m+1 j}^{0}=\delta_{1 j} r_{m+1},
$$

satisfies (4.6). Now since the $(m+1)$ st row, with only one positive element, clearly cannot contribute terms to a positive circuit, $X^{0}$ is also a forest; the theorem is proved.

To apply this method, in the construction of a trial solution, search for the smallest $r_{i_{1}}$ and the largest $c_{j_{1}}$, and then set

$$
x_{i_{1} j_{1}}^{0}=r_{i_{1}}
$$

In effect, this deletes the $i_{1}$ st row, after $c_{j_{1}}$ is replaced by $c_{j_{1}}-r_{i_{1}}$, and the process is repeated (with interchanged rows and columns as necessary) until all $x_{i j}^{0}$ have been determined. For automatic machine calculation, the procedure is easily made unique, for any one starting order of rows and columns, by specifying that the search is first on row-totals when the number of rows is the same as the number of columns at any stage, and that the row-total or column-total with the smallest index is chosen whenever at any stage there are several equal values to choose from. This initial trial solution will be called "preferred" for identification. $^{2}$

THEOREM 4. A trial solution that is a forest of $t$ trees has $m+n-t$ nonzero elements.

Proof. Observe first that if the trial solution $X$ is a forest of $t$ trees, the rows and columns of $X$ can be rearranged so that $X$ has the form

${ }^{2}$ Sometimes, as in this instance, we indicate how to make a unique choice among possible alternatives at each computational step, but usually do not. It is necessary to do this in order completely to routinize the computing steps, of course, but the matter presents no difficulty and we omit it here. 


$$
\left\|\begin{array}{llll}
X_{11} & 0 & \cdots & 0 \\
0 & X_{22} & \ldots & 0 \\
\cdots & \ldots & \cdots \\
0 & 0 & \cdots & X_{t t}
\end{array}\right\|,
$$

where each $X_{a a}$ is a tree. Consequently, the theorem amounts to proving that an $m \times n$ matrix with no zero rows or columns, which is a tree, has $m+n-1$ positive elements. If $m+n=2$, this is obvious, so assume the statement to be true for all matrices for which $m+n=k$ and consider one for which $m+n=k+1$. Since $m \geq n$, clearly some row has only one positive element, as otherwise there would be a positive circuit. Delete this row and apply the induction hypothesis.

In actual cases when $m$ and $n$ are relatively small, or when there is other reason to believe that an initial trial solution better than the preferred one can be found by trial and error, it may be better to construct the initial trial solution in some other way than the one given in the proof of Theorem 3 , in order to reduce the number of steps required in the iterative process.

The methods developed in this paper apply directly for any trial solution that is a forest, and are readily extended for other cases. It is easy to see that there must be at least one solution which is a forest.

8. Nondegenerate case. We consider now the case of a trial solution $X$ which is a tree. Let the positive elements of $X$ be

$$
x_{i_{a} j_{a}} \quad(a=1, \cdots, m+n-1) .
$$

We shall need the following theorem.

THEOREM 5. If $X$ is a trial tree, the set of equations

$$
d_{i j}+u_{i}-v_{j}=0 \text { for }(i, j)=\left(i_{a}, j_{a}\right)
$$

has the general solution

$$
u_{i}=u_{i}^{*}+z, \quad v_{j}=v_{j}^{*}+\iota
$$

where $\left(u_{i}^{*}, v_{j}^{*}\right)$ is a particular solution and $z$ is arbitrary.

Proof. The theorem is apparent for $m=1$, and we proceed by induction. 
Suppose the theorem is true for all trial trees of $m$ rows, and let $X$ be an $(m+1) \times n$ trial tree. Obviously, there must be at least one row of $X$ that has exactly one nonzero element; we may suppose it to be $x_{m+1} n$ without loss of generality - also that

$$
i_{m+n-1}=m+1 \text { and } j_{m+n-1}=n
$$

Since $X$ is a trial tree, the matrix obtained from $X$ by deleting the last row (or, if $m+1=n$, its transpose) is also. The induction hypothesis implies that the general solution of (8.1), with the final equation omitted, is of the form

$$
u_{i}=u_{i}^{*}+z, v_{j}=v_{j}^{*}+z .
$$

We note next that this final equation becomes

$$
u_{m+1}=\left(v_{n}^{*}-d_{m+1 n}\right)+z=u_{m+1}^{*}+z .
$$

The theorem follows easily.

It will be convenient to call the particular solution $\bar{u}_{i}, \bar{v}_{j}$ of $(8.1)$ obtained by setting $u_{1}=0$ the preferred trial solution of the dual problem corresponding to the trial tree $X$. As an obvious consequence of Theorem 5, we state:

COROLLARY 5A. If $X$ is a trial tree, then it is a solution of the problem if and only if the corresponding preferred trial solution $\left(\bar{u}_{i}, \bar{v}_{j}\right)$ of the dual problem satisfies

$$
d_{i j}+\bar{u}_{i}-\bar{v}_{j} \geq 0
$$

for all $i$ and $j$.

All that is needed now in order to establish the method for the nondegenerate case is to show how to construct a new trial matrix $X^{*}$, if $X$ is not a solution, such that

$$
\sum_{i, j}\left(x_{i j}-x_{i j}^{*}\right) d_{i j} \geq 1
$$

In this case, it follows by Corollary $5 \mathrm{~A}$ that

$$
d_{k l}+\bar{u}_{k}-\bar{v}_{l}<0
$$


for at least one pair $(k, l)$ and, of course, $x_{k l}=0$.

THEOREM 6. If the trial solution $X$ is a tree, and $x_{k l}=0$, then there is a unique I-circuit on $X$, where I consists of all positive $x_{i j}$ together with $x_{k l}$.

Proof. It suffices to show that the $l$-graph of $X$ has cyclomatic number $\mu=1$. By assumption, the positive graph of $X$ has cyclomatic number zero; and since $X$ must have positive elements $x_{a l}$ and $x_{k b}$ for some $a$ and $b$, the $I$-graph of $X$ has two more arcs, one more vertex, and the same number (one) of connected pieces. Hence $\mu=1$, and the proof is complete.

Now arrange this unique $I$-circuit $\left[X_{s}\right]$ with $x_{k l}$ as the second term, and let $g$ be the minimum of the odd-terms of $\left[X_{s}\right]$ in this arrangement. If we subtract $g$ from the odd-terms, add $g$ to the even terms, and leave the remaining elements of $X$ unchanged, we get a matrix $X^{*}$ that satisfies (4.6) and is a forest (since $\left[X_{s}\right]$ is unique ).

THEOREM 7. The following relation holds:

$$
\sum_{i, j}\left(x_{i j}-x_{i j}^{*}\right) d_{i j} \geq 1
$$

Proof. Let

$$
\left[X_{s}\right]=\left[x_{i_{1} j_{1}}, x_{i_{1} j_{2}}, x_{i_{2} j_{2}}, x_{i_{2} j_{3}}, \ldots, x_{i_{s} j_{s}}, x_{i_{s} j_{1}}\right],
$$

where

$$
x_{i_{1} j_{2}}=x_{k l} \text {. }
$$

Then

$$
\begin{aligned}
\sum_{i, j}\left(x_{i j}-x_{i j}^{*}\right) d_{i j} & =g\left(d_{i_{1} j_{1}}-d_{i_{1} j_{2}}+d_{i_{2} j_{2}}-d_{i_{2} j_{3}}+\cdots+d_{i_{s} j_{s}}-d_{i_{s} j_{1}}\right) \\
& =-g\left(d_{i_{1} j_{2}}+u_{i_{1}}-v_{j_{2}}\right) \geq i
\end{aligned}
$$

The theorem follows.

If $X^{*}$ is a tree, then the whole process is repeated until at some stage a trial matrix is obtained that either ( $i$ ) is a solution, or (ii) is not a solution 
and is a forest of $t>1$ trees. We shall now discuss (ii).

9. The degenerate case. Let $X$ be a trial matrix which is a forest of $t>1$ trees. As we have seen, we may suppose that the rows and columns of $X$ are ordered so that

$$
X=\left\|\begin{array}{llll}
X_{11} & 0 & \cdots & 0 \\
0 & X_{22} & \cdots & 0 \\
\ldots & \ldots & \cdots & 0 \\
0 & 0 & \cdots & X_{t t}
\end{array}\right\|
$$

where each submatrix $X_{a a}$ of order $m_{a} \times n_{a}$ is a tree. We can apply the methods of the nondegenerate case to the subproblems corresponding to the submatrices $X_{a a}$, and either obtain a solution to each subproblem or further decompose the matrix $X$; thus we may also assume that each $X_{a a}$ is a solution to its subproblem.

By Corollary 5A, we know that

$$
d_{a a}^{i j}+\bar{u}_{a}^{i}-\bar{v}_{a}^{j} \geq 0 \quad\left(a=1, \cdots, t ; i_{a}=1, \cdots, m_{a} ; j_{a}=1, \cdots, n_{a}\right)
$$

where $\bar{u}_{a}, \bar{v}_{a}$ is the preferred trial solution of the dual subproblem corresponding to the solution $X_{a a}$, and that

$$
d_{a a}^{i j}+\bar{u}_{a}^{i}-\bar{v}_{a}^{j}=0 \text { if } x_{a a}^{i j}>0 .
$$

We recall also that the most general values for $u_{a}^{i}$ and $v_{a}^{j}$ are given by

$$
u_{a}^{i}=\bar{u}_{a}^{i}+z_{a}, \quad v_{a}^{j}=\bar{v}_{a}^{j}+z_{a},
$$

where the $z_{a}$ are arbitrary parameters.

It follows from Theorem 2 that $X$ is a solution if and only if there are values of $z_{a}$ that satisfy inequalities corresponding to (4.7), or in our present notation:

$$
d_{a b}^{i}{ }^{j}+u_{a}^{i}-v_{b}^{i} \geq 0 \quad \text { for all } a, b, i_{a} \text {, and } j_{b} .
$$

But (9.3) has a solution for $z_{a}$ if and only if the following inequalities have a solution for $z_{a}$ :

$$
p_{a b}+z_{a}-z_{b} \geq 0
$$


where

$$
\bar{p}_{a b}^{i j}=d_{a b}^{i j}+\bar{u}_{a}^{i}-\bar{v}_{b}^{j}, \quad p_{a b}=\min _{(i, j)}\left\{\bar{p}_{a b}^{i j}\right\}
$$

We have proved:

LEMmA A. The matrix $X$ is a solution if and only if there are real numbers $z_{a}$ such that

$$
p_{a b}+z_{a}-z_{b} \geq 0 \quad(a, b=1, \cdots, t) .
$$

In order to establish a criterion for the solvability of (9.4), we consider a special case of the original problem, defined as follows:

$$
d_{a b}=p_{a b}, r_{a}=c_{b}=1, a, b=1, \cdots, t .
$$

We call this the special problem, the corresponding dual the special dual, and now consider the special combined problem:

$$
\begin{array}{ll}
\sum_{b} y_{a b}=\sum_{a} y_{a b}=1 & \left(y_{a b} \geq 0\right), \\
p_{a b}+z_{a}-w_{b} \geq 0, \quad y_{a b}\left(p_{a b}+z_{a}-w_{b}\right)=0 . &
\end{array}
$$

If we set $y_{a b}=\delta_{a b}$, then for this trial solution the conditions reduce to:

$$
\begin{aligned}
& p_{a b}+z_{a}-w_{b} \geq 0 \quad \text { for } a \neq b, \\
& p_{a a}+z_{a}-w_{a}=0 .
\end{aligned}
$$

Since $p_{a a}=0$, it follows that $z_{a}=w_{a}$, and so these conditions are equivalent to (9.4). Hence, by Theorem 2, (9.4) has a solution if and only if $\left\|\delta_{a b}\right\|$ is a solution of the special problem. Using Lemma A, we now have:

Lemma B. The matrix $X$ is a solution of the original problem if and only if the identity matrix is a solution of the special problem.

THEOREM 8. The matrix $X$ is a solution of the problem if and only if

$$
p_{a_{1} a_{2}} \ldots a_{h} \geq 0 \quad(h=2,3, \cdots, t),
$$


where $\left(a_{1}, a_{2}, \cdots, a_{h}\right)$ is any permutation of $h$ different positive integers, none greater than $t$, and

$$
p_{a_{1} a_{2}} \cdots a_{h}=p_{a_{1} a_{2}}+p_{a_{2} a_{3}}+\cdots+p_{a_{h} a_{1}}
$$

Proof. By Lemma B, it suffices to show that the condition of the theorem is equivalent to the statement that $\left\|\delta_{a b}\right\|$ is a solution of the special problem.

First of all, it is easy to see that at least one solution $Y=\left\|y_{a b}\right\|$ of the special problem is a forest, and hence has less than $2 t$ nonzero elements. That the elements of $Y$ are all either zero or unity can be seen by induction as follows. The basis of the induction is obvious, and we consider the case $t+1$, assuming the statement for $t$. There must be at least one element of $Y$ that is unity, as otherwise $Y$ would have at least $2 t$ nonzero elements. We may suppose that this element is $y_{t+1} t+1$. But then the induction hypothesis implies that each element $y_{a b}(a, b=1, \cdots, t)$ is zero or one. It follows that there are exactly $t$ elements of $Y$ that are unity, whence we can write

$$
\sum_{a, b} y_{a b} p_{a b}=p_{a_{1} b_{1}}+p_{a_{2} b_{2}}+\cdots p_{a_{t} b_{t}},
$$

where $\left(a_{1} a_{2} \cdots a_{t}\right)$ and $\left(b_{1} b_{2} \cdots b_{t}\right)$ are permutations of the first $t$ integers. Then $\left\|\delta_{a b}\right\|$ is a solution of the special problem if and only if always

$$
p_{a_{1} b_{1}}+p_{a_{2} b_{2}}+\cdots+p_{a_{t} b_{t}} \geq p_{11}+p_{22}+\cdots+p_{t t}=0
$$

The proof is completed by noting that this sum can be written as

$$
p_{a_{1} a_{2}}+p_{a_{2} a_{3}}+\cdots+p_{a_{h} a_{1}}
$$

with $\left(a_{1} a_{2} \cdots a_{h}\right)$ as described in the theorem.

We now need to show how to construct an improved trial solution $X^{*}$ in the event that $X$ is not a solution. In this case, we know from Theorem 8 that there is a sum

$$
\vec{p}_{a_{1} a_{2}}^{i^{0} j^{0}}+\bar{p}_{a_{2} a_{3}}^{i^{0} j^{0}}+\cdots+\bar{p}_{a_{h} a_{1}}^{i^{0} j^{0}}<0
$$

Let $I$ consist of all positive elements $x_{a j}^{i j}$ together with all 


$$
x_{a_{k} a_{k+1}{ }^{0}{ }^{0}}
$$

of $X$. Then we assert:

THEOREM 9. There is a unique l-circuit on $X$ that can be arranged to involve as even-terms all the

$$
x_{a_{k} a_{k+1} i^{0}}^{i_{k}}
$$

Proof. The positive graph of $X$ has $m+n-t$ vertices, $m+n-2 t$ arcs, and $t$ connected pieces. Also, for each

$$
x_{a_{k} a_{k+1}^{0} j^{0}}^{i_{k+1}}
$$

there are nonzero elements

$$
x_{a_{k} a_{k}}^{i^{0} j^{1}}, x_{a_{k+1} i^{1}}^{j_{k+1}^{0}}
$$

Hence in passing from the positive graph to the $l$-graph, $h$ vertices and $2 h$ arcs are added, and the number of connected pieces is decreased from $t$ to $t-h+1$. Thus the cyclomatic number of the $l$-graph is

$$
\mu=(2 h+m+n-2 t)-(h+m+n-t)+(t-h+1)=1,
$$

so there is a unique $I$-circuit $\left[X_{s}\right]$ on $X$. Since the graph obtained by omitting from $I$ any

$$
x_{a_{k} a_{k+1}{ }^{0}}^{0}
$$

clearly has no cycle, $\left[X_{s}\right]$ contains all of these.

Evidently $\left[X_{s}\right]$ can be arranged, for example, as

$$
\left[x_{a_{1} a_{1}}^{i^{0} j^{1}}, x_{a_{1} a_{2}}^{i^{0} j^{0}}, x_{a_{2} a_{2}}^{i^{1} j^{0}}, \cdots, x_{a_{2} a_{2}}^{i^{0} j^{1}}, x_{a_{2} a_{3}}^{i^{0} j^{0}}, \cdots\right]
$$

so that all

$$
x_{a_{k} a_{k+1}^{i^{0}}}^{i_{k}}
$$


appear as even-terms.

As in the nondegenerate case, let $g$ be the smallest odd-term in $\left[X_{s}\right]$ (hence $g>0$ ), and define a new trial matrix $X^{*}$ by replacing the elements of $X$ that appear in $\left[X_{s}\right]$ by new ones increased by $g$ for even-terms and decreased by $g$ for odd-terms; the other elements of $X$ are left unchanged. Again $X^{*}$ satisfies the conditions for a trial matrix. To complete the discussion of the degenerate case, it remains only to prove:

THEOREM 10. The follouing relation holds:

$$
\sum_{i, j}\left(x_{i j}-x_{i j}^{*}\right) d_{i j} \geq 1
$$

Proof. Since $X$ and $X^{*}$ differ only on

$$
\left[X_{s}\right]=\left[x_{i_{1} j_{1}}, x_{i_{1} j_{2}}, x_{i_{2} j_{2}}, x_{i_{2} j_{3}}, \ldots, x_{i_{s} j_{s}}, x_{i_{s} j_{1}}\right]
$$

then

$$
\sum_{i, j}\left(x_{i j}-x_{i j}^{*}\right) d_{i j}=-g\left(d_{i_{1} j_{1}}-d_{i_{1} j_{2}}+d_{i_{2} j_{2}}-d_{i_{2} j_{3}}+\cdots+d_{i_{s} j_{s}}-d_{i_{s} j_{1}}\right)
$$

The proof is completed by noting that

$$
d_{i j}=\bar{p}_{i j}+\bar{v}_{j}-\bar{u}_{i} \text { and } \bar{p}_{i j}=0 \text { if } x_{i j}>0
$$

so that

$$
\sum_{i, j}\left(x_{i j}-x_{i j}^{*}\right) d_{i j}=-g\left(p_{a_{1} a_{2}} \cdots a_{h}\right) \geq 1
$$

\section{REFERENCES}

1. Frank L. Hitchcock, The distribution of a product from several sources to numerous localities, j. Math. Physics, 20 (1941), 224-230.

2. L. Kantorovitch, On the translocation of masses, Doklady Akad. Nauk SSSR, 37 (1942), 199-201.

3. Tjalling C. Koopmans, Optimum utilization of the transportation system, Econometrica, 17, Supplement (1949), 136-146. 
4. Activity analysis of production and allocation (Proceedings of the Linear Programming Conference held at the University of Chicago, June, 1949, Tjalling C. Koopmans, Ed., John Wiley and Sons, Inc., New York (1951).
a. Tjalling C. Koopmans and Stanley Reiter, A model of transportation (pp. 222- 259).
b. George B. Dantzig, Application of the simplex method to a transportation problem (pp. 359-373).
c. David Gale, Harold W. Kuhn, and Albert W. Tucker, Linear programming and the theory of games (pp. 317-329).

5. J. M. McLynn and C. B. Tompkins, Application of a duality theorem in the calculation of some linear maximizing and minimizing problems, Engineering Research Associates, Inc. (Unpublished).

6. Alex Orden, A procedure for handling degeneracy in the transportation problem, Headquarters U.S. Air Force, April, 1951 (Unpublished).

7. Julia Robinson, A note on the Hitchcock-Koopmans problem, The RAND Corporation, June 1950 (Unpublished).

8. - On the Hamiltonian game - a traveling salesman problem, The RAND Corporation, December 1949 (Unpublished).

9. Oswald Veblen, Analysis Situs, American Mathematical Society Colloquium Publications, New York (1931).

The RAND Corporation 


\section{PACIFIC JOURNAL OF MATHEMATICS}

\section{EDITORS}

\section{R. U. FOEINSON}

University of California

Berkeley 4, California

E. HewitT

University of Washington

Seattle 5 , Washington
P. P. DILWOR TH

California Institute of Technology

Pasadena 4, California

E. F. BECKENBACH

University of California

Los Angeles 24, California

\section{ASSOCIATE EDITORS}

$\begin{array}{llll}\text { H. BUSEMANN } & \text { P. R. HALMOS } & \text { BØRGE JESSEN } & \text { J. J. STOKER } \\ \text { HERBERT FFDERER } & \text { IIEINZ HOPF } & \text { PAUL LÉVY } & \text { E. G. STRAUS } \\ \text { MARSHALL, IIALI } & \text { R. D. JAMES } & \text { GEORGE PÓLYA } & \text { KÖSAKU YOSIDA }\end{array}$

\section{SPONSORS}

UNIVERSITY OF BRITISH COLUMBIA

CALIFORNIA INSTITUTE OF TECHNOLOGY.

UNIVERSITY OF CAIJIFORNIA, BERKELEY

UNIVERSITY OF CAIJIFORNIA, DAVIS

UNIVERSITY OF CALIFORNIA, LOS ANGELES

UNIVERSITY OF CALIFORNI A, SANTA BARBARA

UNIVERSITY OF NEVADA

OREGON STATE COLLEGE

UNIVERSITY OF OREGON

\author{
UNIVERSITY OF SOU TIERN CALIFORNIA \\ STANFORD RESEARCH INSTITUTE \\ STANFORD UNIVERSITY \\ WASHINGTON STATE COLLEGE \\ UNIVERSITY OF WASHINGTON \\ AMERICAN MATHEMATICAL SOCIETY \\ NATIONAL BUREAU OF STANDARDS, \\ INSTITUTE FOR NUMERICAL ANALYSIS
}

Mathematical papers intended for publication in the Pacific Journal of Mathematics should be typewritten (double spaced), and the author should keep a complete copy. Manuscripts may be sent to any of the editors except Robinson, whose term expires with the completion of the present volume; they might also be sent to M.M. Schiffer, Stanford University, Stanford, California, who is succeeding Robinson. All other communications to the editors should be addressed to the managing editor, E. F. Beckenbach, at the address given above.

Authors are entitled to receive 100 free reprints of their published papers and may obtain additional copies at cost.

The Pacific Journal of Mathematics is published quarterly, in March, June, September, and December. The price per volume (4 numbers) is $\$ 8.00$; single issues, $\$ 2.50$. Special price to individual faculty members of supporting institutions and to individual members of the American Mathematical Society: $\$ 4.00$ per volume; single issues, $\$ 1.25$.

Subscriptions, orders for back numbers, and changes of address should be sent to the publishers, University of California Press, Berkeley 4, California.

Printed at Ann Arbor, Michigan. Entered as second class matter at the Post Office, Berkeley, California.

\section{UNIVERSITY OF CALIFORNIA PRESS • BERKELEY AND LOS ANGELES}




\section{Pacific Journal of Mathematics}

\section{Vol. 3, No. 2 \\ April, 1953}

William George Bade, An operational calculus for operators with spectrum

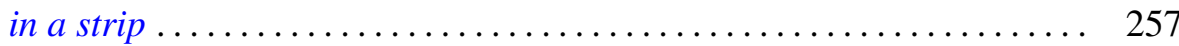

E. F. Beckenbach and Lloyd Kenneth Jackson, Subfunctions of several variables ..................................... 291

David Blackwell, Extension of a renewal theorem ................. 315

L. Carlitz, Some theorems on the Schur derivative ................ 321

Paul Arnold Clement, Generalized convexity and surfaces of negative curvature..................................... 333

Merrill M. Flood, On the Hitchcock distribution problem ............... 369

Watson Bryan Fulks, On the unique determination of solutions of the heat

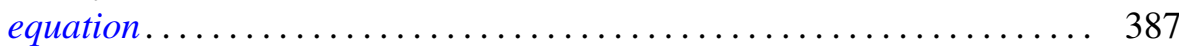

John W. Green, Length and area of a convex curve under affine transformation .................................... 393

William Gustin, An isoperimetric minimax .................. 403

Arthur Eugene Livingston, Some Hausdorff means which exhibit the Gibbs'

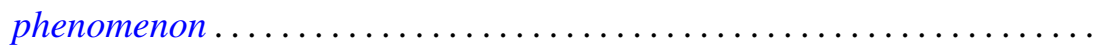

Charles Loewner, On generation of solutions of the biharmonic equation in the plane by conformal mappings ..................... 417

Gábor Szegő, Remark on the preceding paper of Charles Loewner ....... 437

Imanuel Marx and G. Piranian, Lipschitz functions of continuous

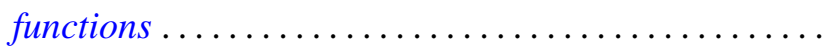

Ting-Kwan Pan, The spherical curvature of a hypersurface in Euclidean space ..

Ruth Lind Potter, On self-adjoint differential equations of second order ...

E. H. Rothe, A note on the Banach spaces of Calkin and Morrey...

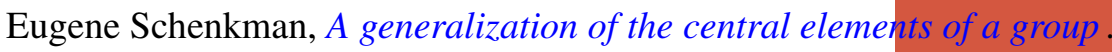

A. Seidenberg, A note on the dimension theory of rings .. . . 